\title{
Osmotic pressure in colloid science: clay dispersions, catanionics, polyelectrolyte complexes and polyelectrolyte multilayers
}

\author{
David Carrière $^{\mathrm{a}, *}$, Miles Page ${ }^{\mathrm{b}}$, Monique Dubois $^{\mathrm{a}}$, Thomas Zemb ${ }^{\mathrm{a}}$, Helmut Cölfen ${ }^{\mathrm{b}}$, \\ Annette Meister ${ }^{\mathrm{c}}$, Luc Belloni ${ }^{\mathrm{a}}$, Monika Schönhoff ${ }^{\mathrm{d}}$, Helmut Möhwald ${ }^{\mathrm{b}}$ \\ a Laboratoire Interdisciplinaire sur l'Organisation Nanométrique et Supramoléculaire (LIONS), \\ CEA/Saclay, F-91191 Gif-sur-Yvette Cedex, France \\ ${ }^{\mathrm{b}}$ Max-Planck Institute for Colloids and Interfaces, D-14424 Golm/Potsdam, Germany \\ ${ }^{\mathrm{c}}$ Martin Luther University Halle-Wittenberg, Institute of Physical Chemistry, Mühlpforte 1, D-06108 Halle, Germany \\ d Institut für Physikalische Chemie, Westfälische Wilhelms-Universität Münster, Corrensstr. 30, D-48149 Münster, Germany
}

\begin{abstract}
Osmotic pressure is a key parameter to understand the thermodynamics and the interactions in colloidal systems. We present here four examples to demonstrate the variety of information that can be extracted from it. The equation of state, i.e. the osmotic pressure versus concentration curve, could be established using analytical ultracentrifugation in binary clay-water mixtures. This method allows a quick and efficient determination of the phase boundaries, and the equation of state shows a good agreement with a Poisson-Boltzmann model. In ternary mixtures of water-anionic surfactant-cationic surfactant, phase separation with a different partitioning of the surfactant in both phases could be evidenced. In the monophasic domains, the surface charge density of the objects could be estimated from the equation of state. In mixtures of polyelectrolytes, different behaviours of the osmotic pressure with respect to the composition could be interpreted in terms of microphase separation, or homogeneous complexation, depending on the composition in polyelectrolyte. Finally, in a colloidal dispersion of spheres coated with polyelectrolytes, three different colloid-colloid interaction regimes could be identified, depending if the polyelectrolyte shells are collapsed onto the colloid, swollen, or non-overlapping. These examples illustrate the variety of information that osmotic pressure can give in a large variety of situations, making this technique an indispensable tool for the physico-chemist.
\end{abstract}

Keywords: Osmotic pressure; Analytical ultracentrifugation; Clays; Catanionic; Polyelectrolyte complexes; Polyelectrolyte multilayers

\section{Introduction}

Osmotic pressure, the derivative of free energy against molar volume, is a thermodynamic variable of universal interest. It is believed to be the driving force for cell expansion [1], controls biological activity [2], and delicate mechanisms regulate the osmotic stress in the intra- and extracellular space [3,4]. Osmotic pressure is also a key parameter in water purification [5] as it controls the flocculation mechanisms through colloidal stability. From a more fundamental point of view, measuring or imposing osmotic pressure is also a tool of choice to study forces between surfaces [6], thus providing a variety of information

\footnotetext{
* Corresponding author. Tel.: +331690854 89; fax: +33169086640. E-mail address: david.carriere@cea.fr (D. Carrière).
}

about the interactions between the surfaces and their environment (ions, solvent, other surfaces). As osmotic pressure is also the chemical activity of the solvent, it is often used specifically for the determination of solvation forces [7].

In this paper, we illustrate the variety of information a physico-chemist can extract from osmotic pressure choosing four particular examples which were handled within the frame of the French-German network "Complex fluids: from 3 to 2 dimensions". First, measuring the osmotic pressure allows determination of boundaries in phase diagrams, and the use of an appropriate method (analytical ultracentrifugation) allows scanning of the complete diagram within a few hours. The second report summarizes how partitioning in aqueous mixtures of surfactants could be detected, by comparing the tie lines with the dilution lines. The third example demonstrates that microphase separated polyelectrolyte complexes can be discriminated from 
homogeneous ones, and gives the fundamental basis for interpretation of the results. Finally, the fourth example deals with one of the most popular applications of osmotic pressure in colloid science, i.e. the measurement of surface forces.

\section{Osmotic pressure measurements in a binary system by analytical ultracentrifugation}

When a coexistence of two phases occurs, the osmotic pressure is expected to be equal in both phases, and to show a plateau independent of composition as long as there is coexistence. Measuring osmotic pressure as a function of composition is therefore a convenient way of detecting boundaries in a phase diagram. This has already been applied for various systems, including the clay-water binary mixture [8]. However, this method usually requires large equilibrium times, i.e. days to weeks. By contrast, analytical ultracentrifugation (AUC) potentially allows scanning of the whole pressure versus composition curve within hours.

The technique consists of measuring the concentration as a function of radial distance in a sample under centrifugation. The sedimentation profile is determined by the variation of osmotic pressure with respect to the position in the sample, i.e. the centrifugal force $[9,10]$ :

$\Delta \Pi\left(r_{i}\right)=\omega^{2}\left(\frac{\partial \rho}{\partial c}\right)_{\mu} \int_{r_{m}}^{r_{i}} c(r) r \mathrm{~d} r$

Here, $r_{m}$ is the distance of the air-water interface from the centre of rotation, $r_{i}$ the radial distance under examination, $\omega$ the angular velocity of centrifugation, $c(r)$ the concentration of the sample at radial distance $r$, and $(\partial \rho / \partial c)_{\mu}$ is the increment of sample density versus concentration at constant chemical potential of low molecular weight species (i.e., in this case, constant ionic strength).

The concentration is determined optically by a refractive index measurement, given by the displacement of interference fringes, $\Delta J$ :

$\Delta J=\frac{\Delta n(r) a}{\lambda}$

where $\Delta n(r)$ is the refractive index change at distance $r$ from some reference point, $a$ the optical pathlength and $\lambda$ is the laser wavelength. $\Delta J$ can thus be converted to $\Delta c$ by knowing the refractive index increment $(\partial n / \partial c)$ of the sample.

Page et al. have used this technique to measure osmotic pressure in laponite-water binary mixtures [11]. This dispersion shows a transition between a dilute isotropic phase and a gel phase, the driving force of which is still under discussion [12-15].

From the sedimentation equilibrium theory, a single-phase system shows an exponential dependence of concentration (and therefore fringe-shift) on the square of the radial distance. Therefore, a plot of $\ln (\phi)$ against $r^{2}$ should yield a straight line in these regions [16]. Fig. 1 shows the raw data from a laponite dispersion with $10^{-3} \mathrm{M}$ salt. Two different linear regimes are detected below concentrations of $1.0 \%$ and above $3 \%$ weight, respectively, attributed to the gel- and dilute-single-phase regions. These values for the phase boundaries correlate well with those

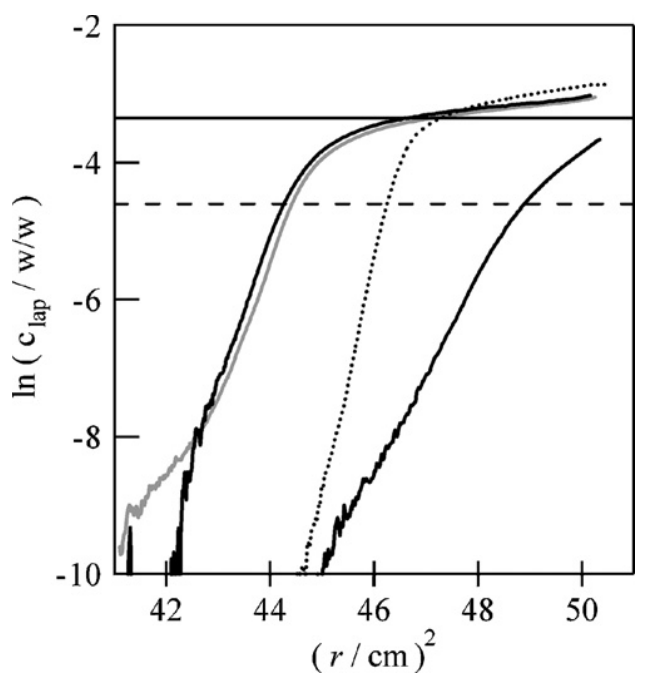

Fig. 1. Plot of $\ln$ (concentration) vs. $r^{2}$ for Laponite samples in sedimentation equilibrium. The various lines correspond to different experimental conditions (initial concentrations, centrifuge rotation speeds and cell dimensions), and gave identical results. Horizontal lines show the dilute (dashed line) and gel (solid line) phase boundaries as determined by Page et al. [11] from Mourchid et al. [17]. The two single-phase regions can be identified by linear sections in the data and are in good agreement with the literature phase boundaries.

published earlier by Mourchid et al. [17], who reported a phase boundary around $2 \%$ weight in the same conditions. Additionally, Page et al. argue that their data support the idea that a two-phase region exists at the intermediate concentrations $(1.0 \%<c<3 \%)$. This may account for the plateau in osmotic pressure measured by others with the usual osmotic stress techniques. The key point in AUC is that the linearity of $\ln (\phi)$ against $r^{2}$ allows an unambiguous determination of what a pure phase is. Finally, the complete reconstruction of the phase diagram by AUC could be performed within some days.

Additionally to the phase boundaries, AUC gives the equation of state, i.e. the osmotic pressure-concentration dependence. The concentration is then usually converted into a mean distance between clay particles (Fig. 2). This allows quantitative comparison with a first-order Poisson-Boltzmann approach, where the colloids are assumed to be plates of infinite area separated by a given water thickness $l_{\mathrm{w}}$ [18]. In the regime where the water thickness is large as compared to the Debye length $1 / \kappa$, the asymptotic form of the Poisson-Boltzmann equation leads to an osmotic pressure equal to:

$\pi=64 k_{\mathrm{b}} T c_{\mathrm{s}}^{\prime} \gamma^{2} \mathrm{e}^{-\kappa l_{\mathrm{w}}}$

where $\gamma \sim 1$ for highly charged membranes and $c_{\mathrm{s}}^{\prime}$ is the salt concentration [19]. When the electrostatic contribution to the osmotic pressure becomes negligible, the osmotic pressure follows the law of Van't Hoff: $\pi=\rho k_{\mathrm{b}} T$ where $\rho$ is the number density of particles.

Fig. 2 shows the good agreement between the equation of state (pressure-distance law) determined experimentally by AUC and the predicted osmotic pressure in both regimes. There are however some discrepancies with the measurements from Mourchid et al. by osmotic stress against dextran solutions [17]. First, no plateau in osmotic pressure could be evidenced around 


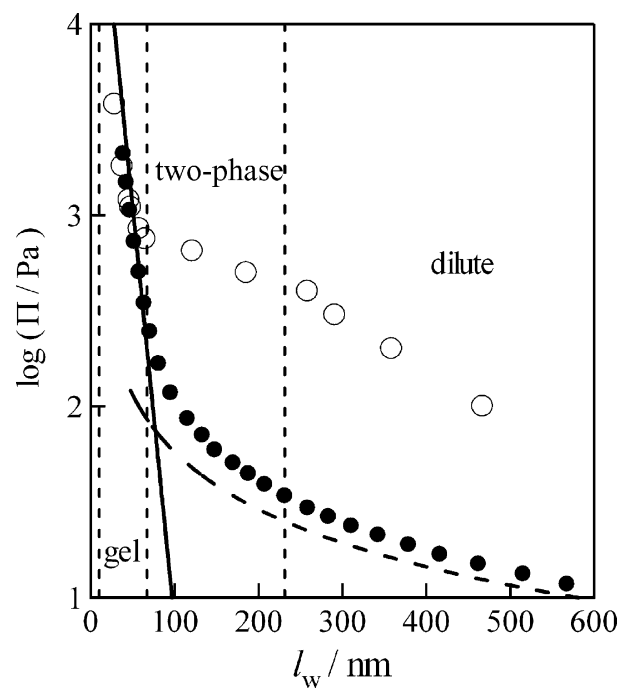

Fig. 2. Osmotic pressure (log scale) plotted against water layer thickness, $l_{\mathrm{w}}$, for disc-like laponite RD particles of thickness $1 \mathrm{~nm}$ and diameter $30 \mathrm{~nm}$. Filled circles: AUC experimental data; open circles: literature experimental data [17]. Solid curve: theoretical osmotic pressure calculated from the asymptotic form of the Poisson-Boltzmann equation; dashed line: theoretical osmotic pressure calculated from the number density of clay particles assuming 'ideal gas' behaviour of the dispersion. Vertical lines delineate the literature-determined phase boundaries at $l_{\mathrm{w}}=67$ and $230 \mathrm{~nm}$, and the Debye length for $10^{-3} \mathrm{M}$ salt of $\sim 10 \mathrm{~nm}$.

the phase transition determined by AUC. This questions the existence of a two-phase region as proposed by Page et al. However, the authors underline one of the peculiarities of the AUC method. No two-phase region can usually be resolved, because for a zero osmotic pressure differential, the entire two-phase region should occur at the same radial distance $r_{i}$ as the two pure-phase boundaries (see Eq. (1)). This should result in a discontinuity in $J$ (from Eq. (2)) at a single-to-biphasic boundary. In reality, the radial distance where any two-phase region should be present is of limited extension, and a flat plateau of pressure cannot be recovered. Of greater curiosity is the discrepancy in the dilute regime between the pressures measured with the two techniques. The authors attributed this variable pressure to different Donnan effect inducing variable salt distributions depending whether the sample is in equilibrium with a reservoir (osmotic stress method) or in a closed system (AUC). Another explanation could be a microphase separation quenching the domain of phase equilibrium. The principle of equality of chemical potentials between phases then becomes:

$\pi_{1}-\pi_{2}=\gamma A$

with $\pi_{1,2}$ the osmotic pressures in both microphases, $\gamma$ an effective interfacial tension between the phases and $A$ is the area of the surface of contact.

\section{Equation of state of catanionic aggregates in the dilute range}

The detection of a plateau in osmotic pressure-composition curves thus usually allows to detect boundaries in phase diagrams. However, in systems with more degrees of freedom than binary mixtures, the thermodynamic response of the system may

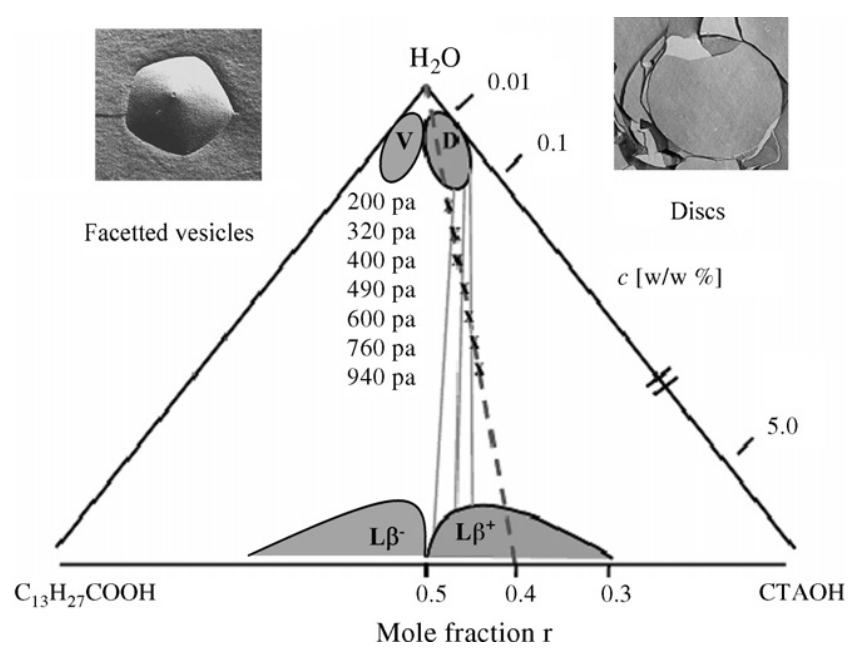

Fig. 3. Phase diagram of the myristic acid/cetyl trimethylammonium hydroxide/water mixture. The osmotic pressures measured along the dilution line are indicated $(x)$. Some tentative tie lines are also indicated.

be more complex. This is for example the case in aqueous mixtures of surfactants of opposite charge, also called "catanionic" mixtures. The large interest in the thermodynamic properties of catanionic assemblies was motivated by their potential use to answer fundamental questions in self-assembly [20,21]. This interest is even broadened by the question of the thermodynamic stability of catanionic vesicles [22,23]. Meister et al. have studied the equation of state of water/myristic acid/cetyl trimethylammonium hydroxide mixtures [24], which is known to produce a variety of morphologies depending on the total surfactant concentration $c$ and the myristic acid molar fraction $r$ : lamellar phases, facetted vesicles or discs [25] (Fig. 3).

In the coexistence domain between lamellar phases and discs, Meister et al. have observed experimentally that the osmotic pressure is not constant upon dilution (Figs. 3 and 4) [24]. This contrasts with the usual expectation that the derivative of osmotic pressure, i.e. the second derivative of free energy, be zero in a coexistence domain. This indicates that volume (i.e. water content) and surfactant compositions are not independent parameters. The osmotic pressure data therefore demonstrate that a different surfactant partitioning occurs in both phases,

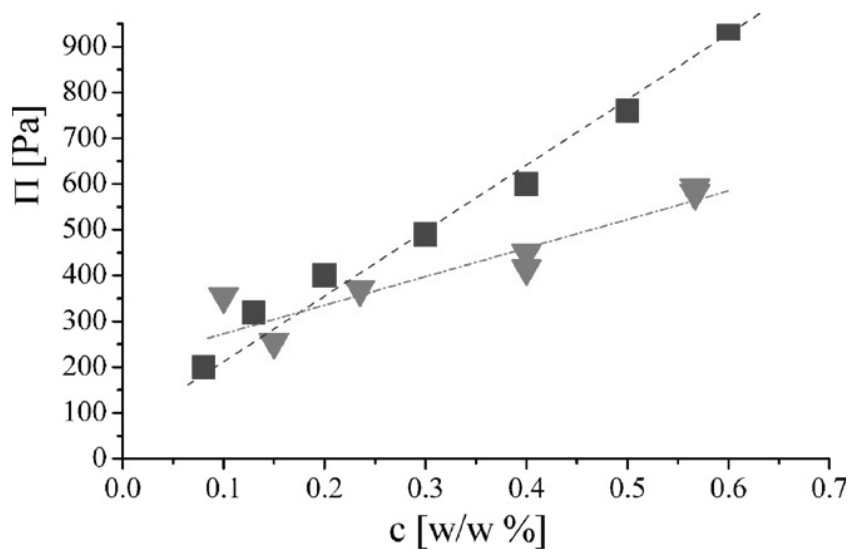

Fig. 4. Osmotic pressure against weight concentration in catanionic mixtures of myristic acid molar fraction $r=0.4$ (square) and $r=0.57$ (triangles). 


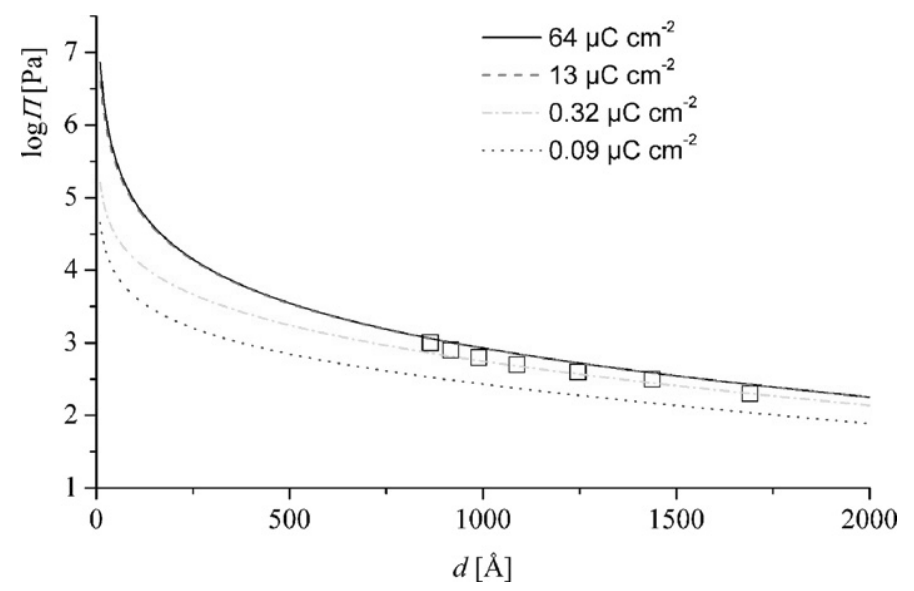

Fig. 5. Comparison between the experimental osmotic pressure and the osmotic pressure between two parallel plates calculated from the Poisson-Boltzmann equation for various surface charge densities. The experimental values are indicated as squares.

with the dilute phase richer in CTAOH than the concentrated phase. Indeed, if the dilution lines of the phase diagram do not coincide with the tie lines a plateau in osmotic pressure is not expected anymore. However, as underlined by the authors, the limit between the $L_{\beta}$ and the disc domain is hard to define, and one may even question if there is any phase coexistence in the thermodynamic sense at intermediate concentrations.

At lower concentrations, the sample enters a monophasic domain that consists either of discs or facetted vesicles, depending on the composition in surfactants. Modeling the objects with simple geometries, i.e. parallel planes, allows comparison of the experimental osmotic pressure with a Poisson-Boltzmann prediction (Fig. 5). Using one single adjustable parameter, the authors found a surface charge density value around $0.3-0.4 \mu \mathrm{C} \mathrm{cm}^{-2}$. However, the estimation of the charge is rough and may vary over two orders of magnitude within the experimental errors achieved. The main result from the study of the equation of state is that the slope of the curve is unambiguously linked to the dimensionality of the swelling: a gas of icosaedralike particles swells in three dimensions, and correlated discs swell in one dimension.

\section{Microphase separation in polyelectrolyte complexes}

Polyelectrolytes of opposite charge are known to form a variety of phases upon mixing, from precipitates of coacervates to stable colloidal complexes [26]. The driving force for this association is the gain in entropy of the counter-ions as two polyeletrolyte segments of opposite charge approach [27]. As a consequence, the measurement of water activity gives a quantitative access to the state of association of the polyelectrolytes. This allowed to demonstrate that the polyelectrolyte segments associate in a 1:1 stoichiometry [28].

In a different approach proposed by Carrière et al. [29], the water activity was measured after removal of the salt released by the complex formation. In this case, the osmotic pressure provides information about the interaction between the complex and the counter-ions of the charged segments in excess.
The results could give information on the microstructure of the polyelectrolyte complexes, depending on their composition. The structural charge concentration of the complex is defined as the excess charge of the complex if the association between polyelectrolytes of opposite charge follows a perfect 1:1 ratio:

$c_{\text {structural }}=1000 \times \frac{|2 f-1|}{f M^{+}+(1-f) M^{-}} c_{\text {weight }}$

where $c_{\text {structural }}$ is the molar concentration of the residual charges $(\mathrm{mol} / \mathrm{kg}) ; c_{\text {weight }}$ the weight concentration of the complex; $M^{+}$ and $M^{-}$the molar masses of the monomers of the polycation and the polyanion, respectively; $f=n^{+} /\left(n^{+}+n^{-}\right)$is the molar fraction in monomers of polycation in the complex.

In the absence of interaction between the counter-ion and the polyelectrolyte complex, the osmotic pressure should be given by the ideal Van't Hoff law:

$\Pi_{\text {ideal }}=c_{\text {structural }} R T$

The osmotic coefficient $K$ measures the deviation of the osmotic pressure from ideality:

$K=\frac{\Pi}{\Pi_{\text {ideal }}}=\frac{c_{\text {effective }}}{c_{\text {structural }}}$

An osmotic coefficient of 1 indicates an ideal behaviour of the counter-ion. A reduction of $K$ indicates a reduction of their activity, and therefore an interaction between the counter-ions and the polyelectrolyte complex.

The osmotic coefficient was measured in complexes of poly(dialyl dimethylammonium chloride) (PDADMAC) and poly(styrene sodium sulfonate) (PSS) of different compositions. At a given molar composition in polyelectrolyte, the osmotic pressure was found to vary linearly with the concentration of complex (Fig. 6). This linear variation results in a constant osmotic coefficient in this range of concentrations, and no variation of the interaction between the residual counter-ions and the complex. As a consequence, the osmotic coefficient can be plotted as a function of the composition of the complex, independently of the concentration (Fig. 7).

The osmotic coefficient was demonstrated to show a highly asymmetric behaviour with respect to the nature of the polyelectrolyte in excess. In excess of polyanion $(f<0.5)$, the osmotic

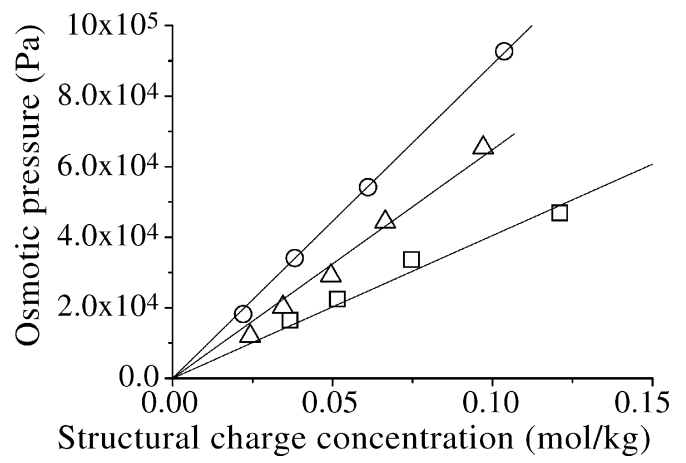

Fig. 6. Osmotic pressure as a function of the concentration of structural charge in complexes of PDADMAC/PSS of molar composition $f=0$ (triangles), $f=0.3$ (circles) and $f=0.7$ (squares) in PDADMAC. The lines are linear fits through the origin. 


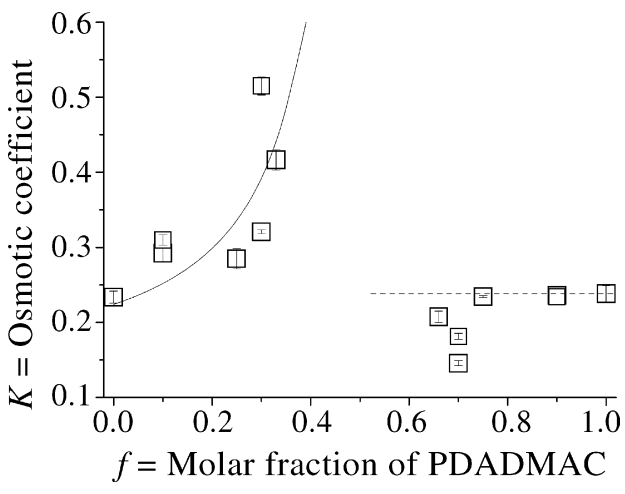

Fig. 7. Osmotic coefficient of the PDADMAC/PSS complexes as a function of the molar fraction of PDADMAC. Full line: prediction for a homogenous complex from a mean-field Manning model (Eq. (11)). Dotted line: prediction for a heterogeneous complex.

coefficient increases with $f$. This shows that the interaction between the residual charges and their respective counter-ions weakens as the charge density of the complex decreases. A simple model based on the estimation of the linear density of the residual negative charges along the PSS chains shows a good agreement with experiments. This model assumes the residual charges in excess to be uniformly distributed along a rod (the PSS backbone). The mean distance $\langle b\rangle$ between charges along this rod is given by:

$\langle b\rangle=b_{0}^{-} \frac{1-f}{1-2 f}$

where $b_{0}^{-}$is the mean distance between charges along the native PSS chain. If the mean separation between charges is above the Bjerrum length $l_{\mathrm{B}}$, the osmotic coefficient of a charged rod is given by [30]:

$K=1-\frac{l_{\mathrm{B}}}{2\langle b\rangle} \quad$ for $\langle b\rangle \geq l_{\mathrm{B}}$

If the mean separation between charges is below the Bjerrum length, the activity of the counter-ions decreases even faster due to ion condensation [30]:

$K=\frac{\langle b\rangle}{2 l_{\mathrm{B}}} \quad$ for $\langle b\rangle \leq l_{\mathrm{B}}$

Finally, this simple model gives an estimate for $K$ using the osmotic coefficient of PSS $\left(K_{0}\right)$ as a single measurable parameter:

$K=K_{0} \frac{1-f}{1-2 f} \quad$ for $K<0.5$,

$K=1-\frac{1}{4 K_{0}} \frac{1-2 f}{1-f} \quad$ for $K>0.5$

For the complexes with excess polyanion $(f<0.5)$, this model captures the main trend of the experimental measurements despite a drastic simplification, namely the assumption that the residual charges are uniformly distributed along a rod. Although no further structural characterization were performed, one can propose that the large persistence length of PSS $(55 \AA$ in the absence of salt [31]) allows this model to depict reasonably well the real situation.

By contrast, in excess of polycation $(f>0.5)$ the condensation of ions onto residual charges fails to capture the experimental data. The osmotic coefficient shows little variation, demonstrating that the interaction between the complex and the counter-ions does not vary with composition, which seems incompatible with a homogeneous complex. However, a constant osmotic coefficient is compatible with a phase separation between the uncompensated positive charges and domains where PDADMAC and PSS neutralize each other. This demonstrates that the polyelectrolyte composition is homogeneous in complexes with excess of PSS, while complexes with an excess of PDADMAC segregates into regions with significantly different PDADMAC compositions.

\section{Interactions between colloids coated by polyelectrolyte multilayers}

A more elaborated system than polyelectrolyte complexes consists in colloidal particles coated by multilayers of polyelectrolytes of opposite charge. Such systems are of particular interest, since removal of the core allows the preparation of hollow polymeric capsules of sub-micron dimensions with a wall thickness on the order of $10 \mathrm{~nm}$ [32]. The stability of the resulting colloidal dispersion depends on the repulsion between the outer shells of the multilayers, about which little is known so far. In a recent article, Dubois et al. reported a full pressure-distance curve in a dispersion of multilayer-coated colloids in the range 1 to $10^{7} \mathrm{~Pa}$ for separation distances between 25 and $6000 \AA$ [33], giving new insight in the mechanisms of stabilization of these colloids.

The osmotic pressure in the colloidal solution was imposed with the osmotic stress technique. The resulting colloid-colloid distance was evaluated from the chemical analysis of residual water. Under the usual assumption that the system is otherwise incompressible, the osmotic pressure is equivalent to a force applied between the colloids [34], thus determining a pressure-distance law, i.e. the equation of state (Fig. 8).

Three distinct regimes could be identified from the full equation of state: at separating distances between the core particles, $2 h$, shorter than $120 \mathrm{~nm}$, the osmotic pressure decays exponentially. This response was attributed to the deformation of the polyelectrolyte multilayers in contact (Fig. 9(1)). In this regime, the compressibility of the layers can be directly evaluated from the isothermal compressibility of the colloid from:

$K_{T}=-\frac{1}{\phi}\left(\frac{\partial \phi}{\partial \Pi}\right)_{T}$

The compressibility modulus of the layers is found to vary between $100 \mathrm{kPa}$ at a distance of $120 \mathrm{~nm}$ up to $1 \mathrm{GPa}$ at the shortest distances. These values are in good agreement with mechanical measurements on hollow polyelectrolyte capsules performed with the colloidal probe technique [35] or with osmotically induced deformations [36].

In the second regime $(120 \mathrm{~nm}<2 h<400 \mathrm{~nm})$, the pressure decays only slightly with increasing distance. This is attributed 


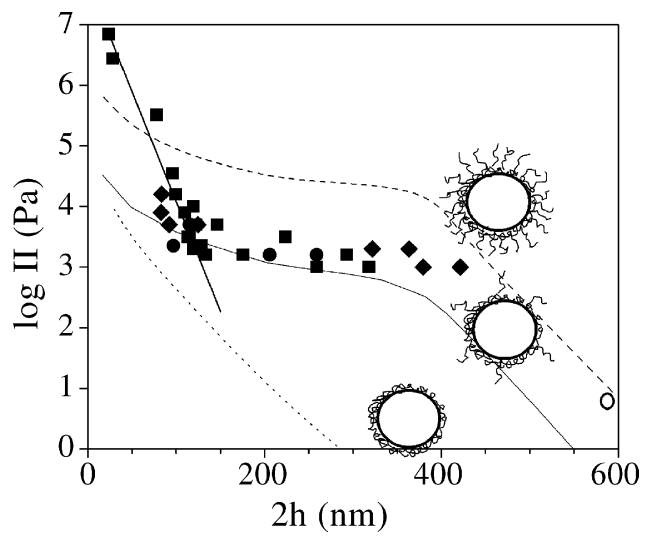

Fig. 8. Equation of state $\log \Pi$ against $2 h$ for multilayer-coated colloids [33]. Different symbols correspond to data points from samples with six or seven layers, respectively, terminated by different polymers which did not lead to differing results. The straight line at small distance is a fit as described in the text for the compression regime. The other lines correspond to PB theories with different geometries for the charged monomer distribution. Dotted line: a total charge $4 \times 10^{5}$ e is collapsed on the particle surface. Dashed line: a total charge $4 \times 10^{5} \mathrm{e}$ is distributed in volume in a brush of layer thickness $200 \mathrm{~nm}$ forming a corona around the particle. Solid line: an adjusted total charge $2 \times 10^{4} \mathrm{e}$ is distributed in volume in a brush of thickness $200 \mathrm{~nm}$.

to an interaction between the extended chains of polyelectrolyte of the terminating layer. These extended chains can be considered as a polyelectrolyte brush (Fig. 9(2)), where the osmotic pressure is governed by the counter-ions. The experimental pressures match well with the prediction from a Poisson-Boltzmann calculation in a spherical geometry, assuming an extension of the brushes up to $200 \mathrm{~nm}$. Interestingly, the modeling showed that these brushes contain only $5 \%$ of the charged segments of polyelectrolyte of the terminating layer, the $95 \%$ others being collapsed onto the particle (Fig. 8, thin full line). A full collapse of the polyelectrolyte, or a full swelling of the brush would lead to an underestimation, or overestimation of the pressure, respectively (Fig. 8, dotted and dashed lines).

For low osmotic pressures $(\Pi<1000 \mathrm{~Pa})$, the system enters a third regime of very weak repulsions. Macroscopically, the particles sediment and the sample shows a sharp meniscus between a concentrated colloidal phase and a clear supernatant. Here, the osmotic pressure cannot be assumed as uniform in the sample, but counterbalances the gravitational forces that cannot be neglected anymore:

$-\frac{\partial \Pi}{\partial z}=\rho(z) m g$

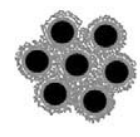

1

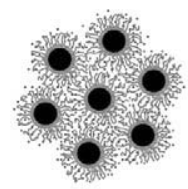

2

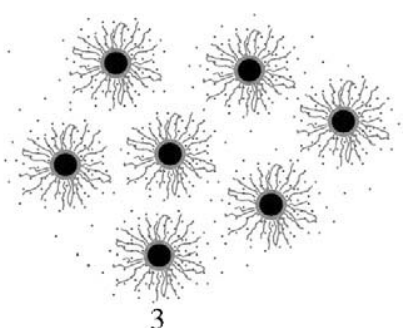

Fig. 9. Sketch of interacting particles in the three different regimes: 1, layer deformation; 2 , swelling brush; 3 , non-overlapping regime. where $\rho$ is the density of particles at the height $z$ and $m$ is the buoyant mass of the particles. As the maximum brush extension is reached with increasing distance (see end of the theoretical curve in Fig. 8), the compressibility is sharply increasing. In this regime the equilibrium of gravitational and colloidal forces leads to an almost constant particle density in the lower phase. The average particle distance $2 h$ corresponds to the maximum brush extension and varies only by a few percent, as calculations showed [25].

The peculiarity of osmotic stress technique allows the equation of state (pressure against distance law) in colloidal systems to be determined over six decades in pressure. For the layer compression regime, comparable experiments were performed with the surface force apparatus in a different geometry, i.e. multilayers deposited on mica sheets [37]. However, osmotic stress is the only technique that allows such a study directly in a colloidal system, on such a large range of pressures. This enabled to reach all three regimes of interparticle interaction.

\section{Conclusion}

These examples demonstrate the variety of uses that osmotic pressure methods can find in colloid science. They range from the most practical applications in formulation, with the determination of boundaries in a phase diagram, to the most fundamental understanding of interactions between complex systems. They also range from the most macroscopic point of view when interpreted in terms of thermodynamics, to a more microscopic view when interpreted in terms of forces or microphase separation. However, when going to such refined views, appropriate modeling is always necessary to make the link between such a macroscopic parameter and a more molecular-level interpretation. Simple Poisson-Boltzmann models have always proven enough for charged systems where the osmotic pressure is dominated by electrostatics. As long as we have not definitely captured different and far more subtle interactions such as hydration forces, dispersion forces, or Hofmeister effects, there will still be a strong need for this intricate combination between modeling and experiments probing interactions between surfaces, ions and solvents.

\section{Acknowledgement}

The authors acknowledge the French-German Network "Complex fluids: from 3 to 2 dimensions" for financial support.

\section{References}

[1] J. Pritchard, New Phytol. 127 (1994) 3.

[2] J. Kornblatt, M. Kornblatt, Biochim. Biophys. Acta: Prot. Struct. Mol. Enzym. 1595 (2002) 30.

[3] B. Kempf, E. Bremer, Arch. Microbiol. 170 (1998) 319.

[4] B. Poolman, J. Spitzer, J. Wood, Biochim. Biophys. Acta: Biomemb. 1666 (2004) 88.

[5] D. Thomas, S. Judd, N. Fawcett, Water Res. 33 (1999) 1579.

[6] P. Claesson, T. Ederth, V. Bergeron, M. Rutland, Adv. Colloid Interf. Sci. 67 (1996) 119.

[7] J. Israelachvili, H. Wennerström, Nature 379 (1996) 219. 
[8] M. Morvan, D. Espinat, R. Vascon, J. Lambard, T. Zemb, Langmuir 10 (1994) 2566

[9] A. Brian, H. Frisch, L. Lerman, Biopolymers 20 (1981) 1305

[10] C. Stanley, H. Strey, Macromolecules 36 (2003) 6888.

[11] M. Page, M. Dubois, T. Zemb, H. Cölfen, Osmotic pressure and phase boundary determination of multiphase systems by analytical ultracentrifugation, submitted for publication.

[12] H. Van Olphen, Discuss. Faraday Soc. 11 (1951) 82.

[13] K. Norrish, Discuss. Faraday Soc. 18 (1954) 120.

[14] H. Tanaka, J. Meunier, D. Bonn, Phys. Rev. E 69 (2004) 031404.

[15] L. Michot, I. Bihannic, K. Porsch, S. Maddi, C. Baravian, J. Mougel, P. Levitz, Langmuir 20 (2004) 10829.

[16] H. Fujita, Foundations of Ultracentrifugal Analysis, Chemical Analysis, vol. 42, John Wiley and Sons, New York, 1975.

[17] A. Mourchid, A. Delville, J. Lambard, E. Lécolier, P. Levitz, Langmuir 11 (1995) 1942.

[18] M. Dubois, T. Zemb, L. Belloni, A. Delville, P. Levitz, R. Setton, J. Chem. Phys. 96 (1992) 2278.

[19] J. Israelachvili, Intermolecular and Surface Forces, Academic Press, London, 1991.

[20] E. Kaler, K. Herrington, A. Murthy, J. Zasadzinski, J. Phys. Chem. 96 (1992) 6698

[21] E. Marques, A. Khan, M. Miguel, B. Lindman, J. Phys. Chem. 97 (1993) 4729.
[22] E. Kaler, A. Murthy, B. Rodriguez, J. Zasadzinski Sci. 245 (1989) 1371.

[23] R. Laughlin, Colloid Surf. A 128 (1997) 27.

[24] A. Meister, M. Dubois, L. Belloni, T. Zemb, Langmuir 19 (2003) 7259.

[25] M. Dubois, V. Lizunov, A. Meister, Th. Gulik-Krzywicki, J.M. Verbavatz, E. Perez, J. Zimmerberg, Th. Zemb, PNAS 101 (2004) 15082.

[26] B. Phillip, H. Dautzenberg, K.J. Linow, J. Kötz, W. Dawydoff, Adv. Polym. Sci. 14 (1989) 91.

[27] A. Veis, J. Phys. Chem. 65 (1963) 1798.

[28] A. Thünemann, M. Müller, H. Dautzenberg, J.F. Joanny, H. Löwen, Adv. Polym. Sci. 166 (2004) 113.

[29] D. Carrière, M. Dubois, M. Schönhoff, T. Zemb, H. Möhwald, PCCP 8 (2006) 3141-3146.

[30] G.S. Manning, J. Chem. Phys. 51 (1969) 924.

[31] E. Dubois, F. Boué, Macromolecules 34 (2001) 3684.

[32] E. Donath, G.B. Sukhorukov, F. Caruso, S.A. Davis, H. Möhwald, Angew. Chem. Int. Ed. 37 (1998) 2202.

[33] M. Dubois, M. Schönhoff, A. Meister, L. Belloni, T. Zemb, H. Möhwald, Phys. Rev. E 74 (2006) 051402.

[34] R. Podgornik, H. Strey, V. Parsegian, Molecular Interactions in Lipids, DNA, and DNA-Lipid Complexes, Marcel Dekker Inc., New York, 2000.

[35] F. Dubreuil, N. Elsner, A. Fery, EPJE: Soft Matter 12 (2003) 215-221.

[36] C. Gao, E. Donath, S. Moya, V. Dudnik, H. Möhwald, EPJE 5 (2001) 21-27.

[37] E. Blomberg, E. Poptoshev, P.M. Claesson, F. Caruso, Langmuir 20 (2004) 5489. 